\title{
El Estudio de la Salud y el Género: \\ Las Ventajas de un Enfoque \\ Antropológico y Feminista (a)
}

\author{
The Study of Health and Gender: \\ The Advantages of an Anthropological and a \\ Feminist Approach
}

Mari Luz Esteban¹

${ }^{1}$ Doctora en antropología social por la Universitat de Barcelona. Profesora titular de antropología social de la Universidad del País VascoEuskal Herriko Unibertsitatea ml.esteban@ehu.es
RESUMEN Como su propio título indica, en este artículo se muestran las ventajas de un enfoque a la vez antropológico y feminista para el estudio de los procesos de salud/enfermedad/atención referidos exclusivamente o no a las relaciones de género. Para ello se analizan distintas cuestiones relacionadas con dos grandes temas: la naturalización social de las mujeres y la distinción de los conceptos de sexo y género. En conjunto, se defiende que un análisis adecuado de las desigualdades en salud de hombres y mujeres requiere una visión no determinista y desnaturalizadora del cuerpo y la salud, así como una utilización correcta del concepto de género, para lo que es preciso tener en cuenta las aportaciones y revisiones feministas, que pueden verse potenciadas por los planteamientos de la antropología de la salud.

PALABRAS CLAVE Sexo; Identidad de Género; Feminismo; Antropología.

ABSTRACT As its title indicates, this article demonstrates the advantages of both an anthropological and a feminist approach to the study of processes relating to health, illness and care whether or not in specific reference to gender relations. To this objetive, different issues are analyzed in relation to two main subjects: the social naturalization of women and the distinction between the concepts of sex and gender. The article as a whole argues that an adequate analysis of the differences in the health of men and women requires a vision which is neither determinist nor denaturalizes the body and health, as well as a correct use of the concept of gender. For this purpose it is important to take into account feminist contributions and revisions, which in turn can be reinforced by the arguments of medical anthropology.

KEY WORDS Sex; Gender Identity; Feminism; Anthropology. 


\section{LOS ESTUDIOS DE SALUD Y GÉNERO: EXPANSIÓN PERO DESCONOCIMIENTO DE LA GENEALOGÍA FEMINISTA}

El estudio de los procesos relativos a las desigualdades de género en salud es un campo de investigación en expansión, tanto en el ámbito de las ciencias biomédicas como sociales. En el marco del Estado Español, en el que trabaja la autora de este artículo, en los últimos años se han multiplicado las reuniones y publicaciones científicas en las que, de un modo específico o no, se exponen este tipo de temáticas (b), y se han ido consolidando las redes de expertas en género (c). Por otra parte, es cada vez más frecuente que sociólogos y antropólogos jóvenes, sobre todo mujeres, se decanten por objetos de estudio relacionados con las diferencias de género, e incluso que investigadores ya consolidados hagan sus primeras incursiones en dicha especialidad.

Este fenómeno tiene que ver con cuestiones muy diversas: la constatación de diferencias en los indicadores de salud de hombres y mujeres, tanto en lo que se refiere a la morbimortalidad y utilización de servicios sanitarios como a los hábitos de salud (d); la presencia creciente de mujeres en la universidad; la búsqueda continua de nuevos objetos de estudio. Pero es también consecuencia de la calidad de las contribuciones, preguntas y críticas feministas, que han desafiado y enriquecido a la ciencia en su conjunto. Sin perder de vista las ventajas que ofrece un campo que, aunque aquejado de cierta periferización, está dotado de apoyos materiales y simbólicos notables, lo que lo convierte en atractivo para investigadores que habían permanecido previamente ajenos al mismo.

Sin embargo, no todas las investigaciones biomédicas o sociales que se refieren a las maneras específicas de enfermar o curarse de hombres y mujeres toman en su justa medida los resultados aportados por los estudios feministas, y mucho menos la evolución en dichas aportaciones. Hoy día sería impensable, por ejemplo, una antropología de la salud y de la medicina que no se hiciera eco de las principales concepciones o discusiones internas a dicha especialidad. Sin embargo, es relativamente habitual leer trabajos donde se abordan materias relativas al género que no integran publicaciones feministas de relevancia, o lo hacen de forma puntual o anecdótica. Este hecho resulta no sólo preocupante por el silenciamiento de los logros y la usurpación de los conocimientos y, por tanto, por la falta de reconocimiento de la producción teórica feminista (2), sino también porque limita y empobrece sobremanera el desarrollo de dichos estudios.

Algunas autoras señalan que existe un problema de "entendimiento" entre feminismo y ciencia, influido por los cuestionamientos profundos que los distintos feminismos han hecho de los principales debates sociales y teóricos (3, 4, 5, $6,7)$. Pero otro factor a tener en cuenta es que muchos autores siguen manteniendo un punto de vista restrictivo y no reciclado a la hora de analizar la estratificación social, dando prioridad a la clase social o a la etnia frente a la exclusión social por género, sin hacer una adecuada articulación de las distintas variables, como reclama la teoría feminista $(8,9)$. Por otra parte, la excesiva compartimentalización del quehacer intelectual en el mundo actual es un obstáculo para que los investigadores se adentren en campos diferentes al suyo. Y una complicación añadida es que las consideraciones feministas son percibidas a veces como una amenaza, en la medida que pueden poner en entredicho los modos de vida y actitudes de los propios investigadores. Pero, sea intencionado o involuntario, el desconocimiento de la bibliografía feminista tiene como consecuencia la utilización ambigua y muchas veces incorrecta del concepto de "género" que, en general, o bien se hace equivalente a "mujer" o bien sustituye de manera mecánica a "sexo". De ahí que muchos estudios simplemente constaten datos referidos a las relaciones entre hombres y mujeres, sin preocuparse por buscar una explicación de las diferencias y desigualdades, es decir, sin intentar aclarar la construcción social que subyace a las mismas (10).

Así y todo, las dificultades comentadas se expresan de diferentes maneras en las ciencias de la salud y en las ciencias sociales. Las/os profesionales de la medicina y enfermería, concienciados o no, tienen impedimentos específicos por su enculturación en una visión esencialista y etnocéntrica del cuerpo y la salud, relacionada a su vez con la propia fundamentación científica de la biomedicina, a la que me referiré posteriormente. 
Pero el ejercicio antropológico, consciente de la necesidad de analizar la experiencia humana considerando los contextos sociales, históricos y culturales en la que se produce, está igualmente afectado por una lectura determinista y esencialista de las mujeres que no se observa en otros temas, y de la que también hablaremos.

El objetivo de este artículo es mostrar algunas ventajas de una mirada antropológica y feminista en el estudio de los procesos de salud/enfermedad/atención. Así, en el abordaje de aspectos que podríamos considerar paradigmáticos en el campo de la salud de las mujeres, como es el caso de la salud reproductiva o la salud mental, es imprescindible tener en cuenta la bibliografía feminista, que es además muy amplia (e). Pero la perspectiva feminista se muestra igualmente útil en campos más generales y/o donde no ha sido aplicada en la misma proporción, como pueden ser las drogas o el sida (f). La posición defendida en este artículo aboga, por tanto, por un diálogo entre disciplinas y perspectivas de análisis que sirva para articular y poner en discusión posiciones y puntos de vista diferentes, desde la idea de que sólo así podremos avanzar en la búsqueda de nuevas preguntas e hipótesis de investigación, y en la identificación de los distintos elementos que hacen que perduren las desigualdades sociales de aquellos grupos que cuentan con menores oportunidades y recursos en función de su clase social, género, etnia u otras variables.

\section{LA SALUD Y EL GÉNERO: NATURALIZACIONES INVISIBLES, DESNATURALIZACIONES NECESARIAS}

Dos ejes centrales del quehacer feminista a lo largo de las últimas décadas, con efectos directos en el ámbito de la salud, han sido la denuncia de la naturalización social de las mujeres, así como la distinción de los conceptos de sexo y género, punto este que trataremos más adelante.

Cuando hablamos de naturalización nos referimos a una percepción absolutamente esencialista y biologicista del cuerpo humano, sobre todo de ciertos cuerpos humanos, que afecta a distintos colectivos (mujeres, negros, homosexuales...), y que lleva implícita su marginación social por razones biológicas y anula toda posibilidad de cambio. En el caso concreto de las mujeres supone teorizar, por ejemplo, que la responsabilización de las mujeres respecto al cuidado de las criaturas o enfermos, una división sexual del trabajo que restringe la igualdad de oportunidades entre hombres y mujeres, se explica por una biología y una psicología diferencial que, en último extremo, se relaciona siempre con la capacidad reproductiva femenina.

Toda una tradición de estudios sociales, históricos y feministas han mostrado que esta argumentación determinista surge en Occidente dentro de un proceso muy amplio de consolidación de un nuevo orden social, económico, político y científico -la sociedad capitalista, burguesa y colonial-, que, aunque desigual y jerárquico, fue presentado como natural y fue legitimado por el evolucionismo social y la noción de progreso. Se constituye así un nuevo sistema de poder y dominación inscrito en el cuerpo y la biología: el biopoder, concepto acuñado por Michel Foucault (15) para poner de manifiesto que el poder no sólo lo ejerce el monarca o el gobierno sino también los discursos expertos que las diversas ciencias vienen desarrollando desde la modernidad; un poder que es experto y productivo a la vez, puesto que actúa a través de establecer las normas de comportamiento. En este contexto, la naturalización, que sigue estando en pleno auge (g), funciona como un subterfugio ideológico totalmente idóneo para resolver las contradicciones surgidas en la sociedades de clases, puesto que intenta aunar la igualdad teórica de oportunidades con las diferencias socioeconómicas y las discriminaciones reales (9).

Si bien las/os historiadoras/es han ido evidenciando la interrelación entre discursos científicos e ideologías sociales y políticas, las antropólogas, por su parte, han denunciado la naturalización implícita al etnocentrismo y determinismo de algunos planteamientos centrales en su disciplina y en las ciencias sociales en general (h). Un etnocentrismo que se ha reflejado, en el plano teórico, tanto en una utilización concreta de conceptos -hogar, familia, parentesco, maternidad, sexualidad, reproducción/producción, doméstico/público- en los que se ha plasmado la 
infravaloración de los valores y costumbres no occidentales, como en la aplicación de modelos interpretativos que implican una clasificación jerárquica de distintas realidades y que legitiman las desigualdades entre poblaciones y grupos sociales (10) (i). Todo esto ha provocado la supervisibilización de los roles reproductivos de las mujeres y la invisibilización de su aportación a la economía, la política o la religión $(4,8,17)$. Las mujeres han sido contempladas como meras reproductoras biológicas; la paternidad ha sido vista como un hecho social y por tanto variable, mientras que la maternidad se ha pensado dependiente de la naturaleza, a partir de la separación absoluta entre lo doméstico y lo público, lo productivo y lo reproductivo (17). En definitiva, la crítica feminista ha hecho un esfuerzo ingente de revisión y desnaturalización del análisis clásico de la reproducción y de la sexualidad, resaltando el carácter dinámico de los procesos reproductivos así como el control social que se ejerce sobre ellos.

Pero, a este respecto, la aportación específica de la antropología al feminismo ha sido mostrar las ventajas de tener en cuenta la diversidad cultural y los riesgos de hacer uniforme y universal la realidad de las mujeres; peligros en los que caen fácilmente algunos discursos con una clara hegemonía dentro del feminismo de la salud, como los que provienen del campo de la medicina y la psicología (19). En este sentido, la antropóloga Nancy ScheperHughes hace en su libro "La muerte sin Ilanto" una crítica contundente a los esquemas médicopsicológicos occidentales en torno a las relaciones "naturales" y las emociones de las madres respecto a sus criaturas, y rechaza las teorías clásicas occidentales del apego maternal que proclaman que si no se da una confianza básica entre madre e hijo/a, puede haber consecuencias negativas en el futuro y que, por tanto, las experiencias emocionales diferentes son antinaturales (14). Una perspectiva que, en definitiva, serviría para desvalorizar y discriminar a las mujeres que no son blancas, de clase media u occidentales. Sin embargo, y a pesar de que sitúa su trabajo en el relativismo cultural, sus reflexiones nos permiten también pensar en la posibilidad de una visión "universalista" de la salud y la cultura. Pero un universalismo alternativo al enfoque aparentemente neutral pero etnocéntrico y de clase de la biomedicina, ya que defiende la búsqueda de esquemas de análisis que propician la posibilidad de recoger todas las experiencias, situaciones y contextos (j).

\section{LAS LIMITACIONES INTRÍNSECAS A LA BIOMEDICINA}

Desde mi punto de vista, los mal Ilamados "sesgos de género", que suelen caracterizar la práctica médico-sanitaria (y también la antropológica), tienen que ver en la mayoría de los casos con la perpetuación de ópticas deterministas y biologicistas de la realidad de las mujeres que suelen permanecer invisibles para los propios profesionales. Pero además, en esta conducta influye también el hecho de que no se perciben como negativas y/o no se replantean en profundidad lo que en antropología de la medicina ha sido definido como presunciones culturales o ideológicas que confieren "científicidad" a la biomedicina, recogidas por Ángel Martínez Hernáez en su artículo "Antropología de la salud. Una aproximación genealógica" (21). Principios directamente relacionados con la visión positivista de la enfermedad que se asienta en Occidente a lo largo del siglo XIX, un fenómeno ampliamente estudiado. Me refiero, en concreto, a la definición médica de la enfermedad como desviación de una norma biológica; la doctrina de que existe una etiología específica de las enfermedades; la noción de que las enfermedades son universales; la idea de que la medicina es neutral; o la dicotomía mente/cuerpo; por citar algunos de los postulados que, a mi parecer, son más influyentes en un diagnóstico generizado de la salud/enfermedad.

Analizar adecuadamente las relaciones entre salud y género implica replantear y encontrar alternativas a estos supuestos. Comenzando por el primero, es fundamental contemplar a un mismo tiempo y de manera articulada las dimensiones biológica, social y cultural de las enfermedades (21), algo por otra parte difícil de Ilevar a cabo. Sin embargo, es bastante habitual, incluso por parte de las especialistas de género, dar por hecho las categorías y diferencias biológicas, e 
intentar relacionarlas luego con las variables sociales o culturales (género, clase, etnia, edad, estilos de vida...), como si éstas fueran algo que se añade y no hubiera un retroalimentación en la forma en que se definen unas y otras. En segundo lugar, es preciso igualmente cuestionar la etiología de las enfermedades tal y como es formulada -como básicamente unicasual- e implementar modelos multicausales para explicar el origen y desarrollo de los distintos malestares. Un tercer principio crucial en la medicina alopática es la idea de que las enfermedades son universales. Algunas comparaciones etnográficas, por ejemplo las realizadas en torno a la menopausia, muestran perfectamente que no hay síntomas universales y que el único aspecto generalizable (en este caso) es el descenso de estrógenos y la desaparición de la menstruación $(12,22)$. En cuarto lugar, abordar en profundidad las desigualdades de género requiere también ir ensayando, como reclama el feminismo y el postestructuralismo, análisis que rompan o al menos pongan en discusión las categorías binarias del pensamiento occidental: mente/cuerpo, hombres/mujeres, masculino/femenino, razón/emoción, cultura/naturaleza... Por último, hay que seguir discutiendo, en la línea de una multitud de trabajos, sobre la supuesta y para muchos deseable neutralidad de la medicina, ya que considero que no es posible una ciencia neutra y que es mejor reconocer y mostrar de partida la situación y parcialidad (23) de cada análisis científico, y promover el debate entre las distintas propuestas y la búsqueda de un conocimiento interdisciplinar y negociado.

En conclusión, poner en cuestión estos principios de la medicina occidental y asumir el particularismo, el relativismo, la sensibilidad a la dimensión histórica y cultural, y las vinculaciones entre lo "natural" y lo "social", como promulga la antropología de la medicina (21), nos puede permitir avanzar en la desnaturalización y desencialización necesaria de los estudios de género. Sólo así podremos impugnar la misma definición y jerarquización de los síntomas y malestares femeninos que se hace desde la biomedicina y que es asumida acríticamente por las ciencias sociales. Una clasificación donde tienen toda la prioridad los esquemas "hormonales" y reproductivistas de la fisiología femenina, y que determinan de manera radical las ideologías médicas y sociales sobre el ser mujer. Esto nos ayudaría a encontrar formulaciones de la salud que no fueran negativas ni siguieran definiendo a las mujeres como las "otras", las "vulnerables", las "patológicas" $(11,24)$. Para ello, las descripciones deberían apoyarse en lecturas de las propias mujeres y hombres, que reflejaran maneras de entender y vivir los padecimientos mucho más diversas y ricas en matices y factores condicionantes (clase social, etnia, edad, experiencia laboral, migración, formas de convivencia, sexualidad...) (24, 25). En resumen, ir adoptando perspectivas que "localicen" perfectamente en cada contexto los síntomas y malestares, sin renunciar a la construcción de modelos explicativos más generales.

\section{SEXO, BIOLOGÍA Y CULTURA: HACIA MODELOS MÉDICO-CIENTÍFICOS NO DICOTOMIZADORES}

Un segundo eje del trabajo feminista que hemos apuntado es la distinción entre los conceptos de sexo y género, en un intento de discernir entre lo que es biología y lo que es cultura. Una separación que ha tenido y tiene un gran potencial científico y político, pero que ha sido al mismo tiempo fuente de ambigüedades y revisiones.

A finales de los años setenta, las feministas anglosajonas comenzaron a definir y divulgar el concepto de género, que proviene del término inglés gender (k); de esta forma, propusieron utilizar el término "sexo" para designar las diferencias físicas, anatómicas y fisiológicas de hombres y mujeres, que se asocian a su capacidad de procreación, mientras que "género" suponía un nivel de abstracción distinto, mediante el cual se aludía a la jerarquización de espacios y funciones sociales y la diferenciación en el acceso al poder implícitas en las ideas, representaciones y prácticas de hombres y mujeres. Esta nueva noción permitía, por tanto, hacer una crítica política "de las convicciones bio-ideológicas occidentales acerca de la subordinación de las mujeres" (26). Pero, a nivel general, esta diferenciación entre sexo y género ha comportado algunos problemas que han ido siendo resueltos por las feministas (I). Uno de los principales es que se ha 
afianzado la idea de que el sexo (biología) es algo dado, estático, invariable, desde un esquema general de lo biológico como esencial, inamovible, que está siendo revisado en la actualidad; y de que el género (cultura) es lo que se construye, lo que se moldea socialmente. $Y$ en muchos trabajos de investigación se parte de la base de que las diferencias sexuales, e incluso la sexualidad, son "hechos naturales".

En este sentido, desde la antropología feminista -social y física-, la historia y algunos sectores médicos feministas, se está subrayando el carácter de construcción cultural e histórica de la noción de sexo dominante en nuestra sociedad (28-35). Una contribución valiosa de la antropología ha sido mostrar que las gramáticas sexuales, las formas de hablar de las diferencias sexuales, son locales, culturales, no universales (31), ya que surgen en sistemas sociales, de género y de parentesco concretos y distintos. Es decir, que las conceptualizaciones y teorizaciones científicas no son nunca disociables de las concepciones culturales acerca de las relaciones y jerarquías sociales. Así, no pueden ser semejantes, por ejemplo, la interpretación de la fisiología reproductiva de una sociedad matrilineal, donde la contribución masculina a la procreación se considera irrelevante, como sucede en las islas Trobriand de Melanesia (36), a las teorías de una sociedad absolutamente patriarcal como la de los Baruya de Nueva Guinea, donde el semen es visto como principio vital por excelencia (37). Dicho de otro modo, que son las desigualdades sociales -el género- las que interpretan y explican las diferencias biológicas -el sexo- de una determinada manera, y no al revés, como se piensa habitualmente.

En concreto, el concepto occidental de sexo se ha construido y se construye desde una mirada totalmente jerárquica, dicotómica y heterosexual del cuerpo humano y de las relaciones sexuales (32-34). Y este "modelo de dos sexos" (28) condiciona la manera de percibirnos a nosotros mismos y de percibir social y médicamente a hombres y mujeres, y viceversa; todo ello al margen de que la experiencia individual de la identidad de género sea mucho más diversa, híbrida y contradictoria.

Entonces, ¿qué tipo de ciencia correspondería a una sociedad igualitaria entre hombres y mujeres? A mi entender, una ciencia que ensayara modelos alternativos y no dicotómicos para explicar el sexo y la diferencia sexual y humana, como los propuestos ya por algunas científicas (32-34). Además, una práctica científica "de la diversidad" que no contemplara "la" diferencia sexual como un principio absoluto e inmutable, sino como una hipótesis de trabajo a demostrar (16), que relacionara adecuadamente los aspectos biológicos y los sociales, y que profundizara en las diferencias pero también en las similitudes entre hombres y mujeres en cada contexto (25).

\section{EL GÉNERO ES LO QUE SE HACE: PRÁCTICAS CORPORALES, SOCIALES E INSTITUCIONALES}

Respecto al concepto de género, una dificultad manifiesta es que, a pesar de que se define como una construcción cultural, se convierte habitualmente en un concepto ahistórico y acrítico, configurador de identidades tanto o más esencialistas que las producidas por la biología (23). Además de los problemas comentados más arriba, de equiparar género a "mujer" o sustituir sexo por género, frecuentemente se utiliza también un concepto de género dualizado (femenino/masculino), como el de sexo, sin tener en cuenta su dimensión relacional, el cómo se constituye y se retroalimenta socialmente lo femenino y lo masculino de manera dinámica, así como las diferencias en las realidades e intereses dentro de los colectivos masculinos y femeninos (38-41).

Hemos tratado anteriormente el tema de la naturalización de las mujeres. Pero coincido con Will Courtenay en que la salud de los hombres está también de alguna manera naturalizada e invisibilizada, ya que sus especificidades no están suficientemente estudiadas ni explicadas y se toman como naturales e inevitables (41, 42) (m). Este autor defiende que sólo una perspectiva constructivista, relacional y feminista de la salud nos permitirá entender que la mayor mortalidad o siniestralidad de los hombres, o el que éstos acudan menos a centros de atención primaria, por citar algunos ejemplos, tienen que ver con unas concepciones y comportamientos concretos respecto a la salud, asociados a la 
forma en que los hombres se construyen como tales y se presentan socialmente como dominantes. En este sentido, da mucha importancia a las prácticas de riesgo asumidas mucho más por hombres que por mujeres -consumo de sustancias, formas de conducción, expresiones de violencia...-, ya que para él la masculinidad es definida en oposición a los hábitos de vida saludables; una masculinidad que utiliza el cuerpo y la genitalidad como expresión de virilidad y hombría (43). Sin embargo, son estas mismas ideas y conductas las que les permitirían a los hombres acceder a un poder y un prestigio que no tienen las mujeres, aunque sea en grados y formas variables de acuerdo a su clase social, etnia, etc. Además, si bien estas prácticas son ejecutadas entre hombres y entre hombres y mujeres, están apoyadas y sancionadas por instituciones concretas: ejército, empresa, sistema judicial, industria del deporte, sistema sanitario, medios de comunicación... Pero este tratamiento social de los problemas de salud de los hombres no sería más que la otra cara de la moneda de un discurso médico-científico que define los cuerpos femeninos como deficientes (41).

En los años noventa se da un paso más en la re-definición del concepto de género, ya que de la confluencia del anti-esencialismo y de la Ilamada teoría social de la práctica -que privilegia el estudio de la agency, la acción social e individual-, surgen nuevas teorías, como la performativa (26). Así, autoras como Judith Butler desafían la noción estática de la identidad de género y abordan de manera alternativa el proceso de definirse como mujer u hombre (44). El género es, para Butler, efecto de un conjunto de normas y prácticas que regulan la identidad e imponen un modelo de heterosexualidad obligatoria, pero las identidades femenina y masculina no son ni uniformes ni estables, "sino que se encuentran en un permanente proceso de construcción y, por consiguiente, pueden ser resignificadas (...) El género se convierte en algo que se hace en vez de ser algo que se es" (26).

En mi último trabajo, un estudio corporal de las transformaciones en las identidades y prácticas de género (45), me hago eco de las ideas de Butler y de Robert Connell (46), para defender que ser hombre o mujer es algo que se va configurando no sólo a partir de unos actos, discursos y representaciones simbólicas, sino que tiene una base reflexivo-corporal, material, física, performativa; es decir, que la identidad está absolutamente encarnada. Considero que las aproximaciones actuales de la antropología y teoría social del cuerpo están abriendo el camino a un análisis de la experiencia humana, del género y de la salud no biologicista pero tampoco totalmente constructivista, que incorpora la experiencia corporal reflexiva (46) y que permite definir de forma amplia y compleja los circuitos establecidos entre cuerpos, ideologías, relaciones sociales e instituciones (n).

\section{EL GÉNERO COMO UN "HECHO SOCIAL TOTAL": EL ESTUDIO DE LOS CUIDADOS DE SALUD}

Uno de los argumentos centrales en este artículo, como se ha señalado en la introducción, es que una antropología de la salud que integre a la vez la perspectiva feminista permite un análisis de la realidad que puede trascender el estudio concreto de las diferencias entre hombres y mujeres. E incluso el análisis de los procesos de salud/enfermedad/atención, añadimos ahora. Un aspecto que puede ayudarnos a mostrar esto último es el de la atención socio-sanitaria a lo que está denominando la "dependencia", es decir, el cuidado de las personas que por razones de edad o enfermedad no pueden valerse por sí mismas. Un tema de mucha trascendencia en este momento histórico en Europa, puesto que la situación social se ha modificado de manera muy significativa, por los cambios demográficos y sanitarios ocurridos (aumento de enfermedades crónicas y de la esperanza de vida), la nueva situación social y laboral de las mujeres, y las transformaciones en las formas de convivencia. Este nuevo escenario ha llevado a las instituciones a la implementación de políticas públicas diversas en torno a la denominada conciliación entre la vida familiar y laboral (47).

Los datos numéricos no dejan lugar a dudas: en el ámbito español, tres cuartas partes de estos cuidados son desempeñados por las familias y, dentro de ellas, en una proporción similar, por las mujeres adultas $(48,49)$. Es decir, 
hoy día, el trabajo de cuidar es una responsabilidad social absolutamente generizada, pero es una actividad que, sin embargo, no está bien visibilizada y reconocida socialmente, debido fundamentalmente a tres factores:

1) al hecho de que sea la familia la principal institución donde se llevan a cabo, quedando asociadas estas funciones al ámbito de "lo privado";

2) a su difícil catalogación como trabajo, por el componente afectivo y elevado contenido moral, no reconociéndose como actividades que suponen tiempo y dedicación y requieren un conjunto de saberes y técnicas aprendidas a lo largo de toda la vida;

3) a la fuerte naturalización que sufren, de forma que se piensa que las mujeres por el hecho de serlo poseen naturalmente esos saberes y habilidades, sin verlo como consecuencia directa de una división sexual concreta del trabajo dentro de un determinado sistema de género (50).

Es además un campo de investigación que enlaza directamente con uno de los núcleos temáticos de la antropología de la salud, el estudio de la autoatención o sistema "doméstico" de atención (o). La antropología de la medicina, por sus planteamientos teórico-metodológicos y su mirada cualitativa y relativista, permite acceder de una manera más equitativa y compleja a las tareas de atención a la salud dentro y fuera del hogar, ya que toma en cuenta y equipara de entrada los diferentes sistemas médicos y niveles de atención que concurren en cualquier itinerario asistencial (55).

Algunas feministas están Ilamando la atención sobre el alto potencial de crítica y cuestionamiento del sistema socioeconómico que existe alrededor de lo que denominan la "crisis de los cuidados" que sufre la sociedad occidental $(56,57)$. Ellas señalan que, sin embargo, esto suele quedar a veces camuflado por la propia terminología utilizada en los debates (cuidados ( $p$ ), dependencia, vejez, mujeres...), que hace que se perciba como algo que afecta a una población aparentemente concreta y marginal (57). Sin embargo, argumentan, los problemas de conciliación y atención a la dependencia que se están dando, se están produciendo al haber quebrado el modelo previo de cobertura y ponerse de manifiesto las tensiones antes ocultas por las contradicciones entre la acumulación de capital y la sostenibilidad de la vida; de forma que ahora se tiende a la mercantilización y privatización del cuidado al no querer hacerse cargo del mismo ni el colectivo masculino ni el estado, lo que ellas denominan un cierre reaccionario de la crisis.

Considero que este tipo de análisis son del máximo interés, pero que pueden verse enriquecidos por los conceptos económicos, políticos y jurídicos utilizados en el estudio etnográfico actual de los sistemas de reciprocidad e intercambio, los sistemas de parentesco o la división social del trabajo, más poderosos y transculturales. Pero, en todo caso, este ejemplo nos sirve para mostrar cómo el análisis feminista de problemas sociales que aparentemente sólo afectan a las mujeres y a colectivos que suelen quedar al margen de lo definido socialmente como "lo importante", evidencia procesos económicos y políticos globales de cambio de mucha trascendencia para la humanidad. Es decir, que el género puede ser considerado como un "hecho social total" (58) que tiene, por tanto, que ser analizado desde las diversas dimensiones -economía, política, salud, religión...- y que sirve para explicar la cultura en su conjunto $(59,60)$.

A modo de conclusión, en este artículo he tratado de mostrar las utilidades para el estudio de la salud de un enfoque feminista, interdisciplinar, desnaturalizador, no dualista y crítico con los esquemas científicos occidentales. Asimismo, he defendido el gran potencial del concepto de género, entendido como un "principio de organización social" (60), que nos permite niveles y dimensiones de análisis muy diferentes, tanto de las desigualdades en la salud de hombres y mujeres como de fenómenos sociales más amplios. 


\section{NOTAS FINALES}

a. Agradezco a Margaret Bullen, Carmen Díez Mintegui, Rosa Medina Doménech y Mari Jose Peleteiro sus aportaciones y sugerencias a este artículo.

b. Véase, por ejemplo, el VI Informe SESPAS de la Sociedad Española de Salud Pública y Administración Sanitaria, de mayo de 2004, que lleva por título "La salud pública desde la perspectiva de género y clase social" (1), y que puede consultarse en la siguiente dirección de internet: www.sespas.es/infor.html

c. Una de las más importantes es la Red de Mujeres Profesionales de la Salud, coordinada por el Programa "Dona y Salut" (Mujer y Salud) del CAPS-Centre d'Analisis i Programes Sanitaris de Barcelona, que celebra un encuentro anual y sirve además de espacio de intercambio cotidiano entre sus participantes, y a la que pertenece la autora de este artículo.

d. Los hombres tienen una mortalidad mayor y una menor esperanza de vida, y hombres $y$ mujeres presentan un morbilidad diferencial en distintos campos (salud reproductiva, mental, laboral...). Las razones para ello no están del todo claras: se suele hablar de factores biológicos y ambientales, pero la mayor o menor prioridad dada a unos o a otros suele depender del marco teórico de cada estudio. En mi opinión, hay que ser cautos a la hora de hacer lecturas excesivamente diferenciadas por sexo. Por ejemplo, algunas encuestas de salud del ámbito español dejan entrever que, respecto a lo que se denomina la salud percibida, las diferencias por clase social serían más importantes que las diferencias por sexo; por otra parte, en algunos hábitos de salud, como el consumo de alcohol y tabaco, parece haber una influencia del sexo pero también de la edad.

e. Algunas antrópologas de referencia obligada en el estudio de la salud y el género son: Emily Martin (11), Margaret Lock (12), Rayna Rapp (13) o Nancy Scheper-Hughes (14).

f. En la antropología de la salud del Estado Español, las temáticas en las que la perspectiva de género está más consolidada son: la salud reproductiva, los trastornos de la alimentación, y el uso de drogas y fármacos. La mayoría de las investigadoras están vinculadas al programa de doctorado de antropología de la medicina de la Universitat Rovira i Virgili de Tarragona.

g. En la última década del siglo $X X$ se produce una nueva oleada de naturalización, que se proyecta en temas como la menopausia o la maternidad, proceso que he denominado de re-naturalización (16). h. Algunas autoras que han recopilado la crítica y el trabajo de las antropólogas feministas son: Nicole-Claude Mathieu (4), Britt-Marie Thurén (5), Henrietta Moore (8), Virginia Maquieira (10) y Susana Narotzky (17).

i. A nivel empírico, antropólogas como NicoleClaude Matthieu (4) o Paola Tabet (18) han subrayado que las diferentes "tareas reproductivas" -embarazo, parto, lactancia, y cuidados de las criaturas, etc.- no han sido apenas estudiadas en la disciplina por no ser consideradas prácticas culturales.

j. Por ejemplo, cuando se refiere al establecimiento del vínculo materno, defiende la idea de que podría existir una "fase" previa al reconocimiento de la criatura como ser humano, que María Piers ha denominado de "extrañamiento básico" (20), así como la de que todo recién nacido necesita ser "adoptado".

k. Este término fue utilizado por primera vez en los años cincuenta por los médicos y psicólogos que atendían casos de transexualidad e intersexualidad.

I. Un análisis del proceso de definición y revisión del género puede encontrarse en el artículo "La mujer es puro cuento: la cultura del género", de Verena Stolcke (26); así como en el capítulo primero del libro "Modelos emergentes en los sistemas y las relaciones de género", de Teresa del Valle y cols (27).

m. Su trabajo se refiere a Norteamérica pero muchas de sus ideas pueden generalizarse a todo Occidente.

n. Con todo esto no se está planteando la imposibilidad de utilizar las categorías "género", "mujeres", "hombres", ya que es evidente que en Occidente sigue operando una distinción social reflejada en representaciones, símbolos, expectativas, asignación de esferas y funciones sociales. Se trataría más bien de introducir las reflexiones, interrogantes y modificaciones necesarias que nos permitan unos diagnósticos de la salud/enfermedad menos lineales y simplistas (6).

o. A este respecto, son referencia obligada las aportaciones teóricas de Eduardo Menéndez (51, 52), así como los estudios realizados en México por las antropólogas Lilián González Chévez y Rosa María Osorio, que han abordado distintos aspectos de la atención a la salud ofertada por mujeres de clase baja, mostrando el papel fundamental y autónomo de las mujeres en el mantenimiento de la salud de la población $(53,54)$.

p. La misma distinción entre cuidar y curar resulta hoy día problemática, por la dificultad de establecer unas fronteras claras entre una y otra, y por la jerarquización que implica entre tareas y entre profesiones sanitarias. 


\section{REFERENCIAS BIBLIOGRÁFICAS}

1. Informe SESPAS 2004. La salud pública desde la perspectiva de género y clase social. Gaceta Sanitaria. 2004;18 (Supl 1).

2. Del Valle T. Identidad, memoria y juegos de poder. DEVA. 1995;2:14-21.

3. Scott SW. El género: una categoría útil para el análisis histórico. En: Amelang JS, Nash M, editores. Historia y Género: Las mujeres en la Europa Moderna y Contemporánea. Valencia: Edicions Alfons el Magnànim-Institució Valenciana d'Estudis i Investigació; 1990. p. 23-56.

4. Mathieu NC. L'Anatomie politique. Catégorisations et idéologies du sexe. París: Côtefemmes éditions; 1991.

5. Thurén BM. El poder generizado. El desarrollo de la antropología feminista. Madrid: Instituto de Investigaciones Feministas, Universidad Complutense de Madrid; 1993.

6. Esteban ML, Díez Mintegui C. Introducción. En: Esteban ML, Díez Mintegui C, coordinadores. Antropología feminista: desafíos teóricos y metodológicos. Ankulegi-Revista de Antropología Social.1999:9-28.

7. Del Valle T. Introducción. En: Del Valle T, editora. Perspectivas feministas desde la antropología social. Barcelona: Ariel; 2000. p. 9-24.

8. Moore HL. Antropología y feminismo. Madrid: Editorial Cátedra-Feminismos; 1991.

9. Stolcke V. ¿Es el sexo para el género como la raza para la etnicidad? Mientras Tanto. 1992; 48:87-111.

10. Maquieira V. Género, diferencia y desigualdad. En: Beltrán E, Maquieira V, editores. Feminismos. Debates teóricos contemporáneos. Madrid: Alianza editorial; 2001. p. 127-190.

11. Martin E. The Woman in the Body. Boston: Beacon Press; 1987.

12. Lock M. Encounters with Aging: Mythologies of Menopause in Japan and North America. Berkeley: University of California Press; 1993.

13. Rapp R. Testing Women, Testing the Fetus: The Social Impact of Amniocentesis in America. New York: Routledge; 1999.

14. Scheper-Hughes N. La muerte sin Ilanto. Violencia y vida cotidiana en Brasil. Barcelona: Ariel Antropología; 1997.

15. Foucault M. Vigilar y castigar. México: Siglo $\mathrm{XXI} ; 1981$.
16. Esteban ML. Feminismo y salud: balance de tres décadas. En: Seminario Balance y perspectivas de los estudios de las mujeres y del género. Madrid: Instituto de la Mujer. p. 247-257. (Serie Debate; vol 40).

17. Narotzky S. Mujer, Mujeres, Género. Una aproximación crítica al estudio de las mujeres en las Ciencias Sociales. Madrid: Consejo Superior de Investigaciones Científicas; 1995.

18. Tabet P. Fertilité naturelle, reproduction forcée. En: Mathieu NC, compilador. L'arraisonnement des femmes. Essais en anthropologie des sexes. Paris: Éditions de l'École des Hautes Études en Sciences Sociales; 1985. p. 61-146.

19. Esteban ML. La maternidad como cultura: algunas cuestiones sobre lactancia materna y cuidado infantil. En: Perdiguero E, Comelles JM, editores. Medicina y cultura. Estudios entre la antropología y la medicina. Barcelona: Edicions Bellaterra; 2000. p. 207-226.

20. Piers M. Infanticide. New York: Wiley; 1978.

21. Martínez Hernáez A. Antropología de la Salud. Una aproximación genealógica. En: Prat J, Martínez Hernáez A, editores. Ensayos de antropología cultural. Barcelona: Ariel Antropología; 1996. p. 369-381.

22. Hunt K. ¿Una cura para todas las Enfermedades? Interpretación de la menopausia y las complicaciones del tratamiento hormonal sustitutivo. En: Wilkinson S, Kitzinger C. Mujer y salud. Una perspectiva feminista. Madrid: Instituto de la Mujer; 1996. p. 155-180.

23. Haraway D. Ciencia, cyborgs y mujeres. La reinvención de la naturaleza. Madrid: CátedraFeminismos; 1995.

24. Esteban ML. Re-producción del cuerpo femenino. Discursos y prácticas acerca de la salud. Donostia-San Sebastián: Gakoa-Tercera Prensa; 2001.

25. Esteban ML. El género como categoría analítica. Revisiones y aplicaciones a la salud. En: Miqueo $\mathrm{C}$ et al., editores. Perspectivas de género en salud. Madrid: Minerva; 2001. p. 25-52.

26. Stolcke V. La mujer es puro cuento: la cultura del género. Quaderns de I'Institut Catalá d'Antropologia. 2003;19:69-95.

27. Del Valle $T$, coordinadora. Apaolaza JM, Arbe F, Cucó J, Díez C, Esteban ML, Etxeberria F, Maquieira V. Modelos emergentes en los sistemas y las relaciones de género. Madrid: Narcea; 2002.

28. Laqueur T. La construcción del sexo. Madrid: Cátedra-Feminismos; 1994. 
29. Peyre E, Wiels J, Fonton M. Sexe biologique et sexe social. En: Hurtig MC, Kail M, Rouch H, editores. Sexe et genre. De la hiérarchie entre les sexes. París: Editions du Centre National de la Recherche Scientifique; 1991. p. 27-50.

30. Herdt G, editor. Third Sex. Third Gender. Beyond Sexual Dimorphism in Culture and History. New York: Zone Books; 1996.

31. Mathieu NC. Les transgressions du sexe et du genre à la lumière de données ethnographiques. En: Hurtig MC, Kail M, Rouch H. Sexe et genre. De la hiérarchie entre les sexes. París: Centre National de la Recherche scientifique; 1991. p. 69-80.

32. Nieto JA, editor. Transexualidad, transgenerismo y cultura. Antropología, identidad y género. Madrid: Talasa; 1998.

33. Fausto-Sterling A. Sexing the body: Gender Politics and the Construction of Sexuality. New York: Basic Books; 2000.

34. Barral Morán MJ. Genes, género y cultura. En:. Miqueo C. et al. Perspectivas de género en salud. Fundamentos científicos y socioprofesionales de diferencias sexuales no previstas. Madrid: Minerva ediciones; 2001. p. 135-162.

35. Cleminson $R$, Medina Doménech $R$. Hermafroditismo, medicina e identidad sexual en España, 1860-1925. Dynamis. 2004;24:53-91.

36. Malinowski B. La vida sexual de los salvajes del nordeste de la Melanesia. 3a ed. Madrid: Ediciones Morata; 1975.

37. Godelier M. La producción de grandes hombres: Poder y dominación masculina entre los Baruya de Nueva Guinea. Madrid: Akal Universitaria; 1986.

38. Stolcke V. Antropología del género. En: Prat J, Martínez Hernáez A, editores. Ensayos de Antropología Cultural. Barcelona: Ariel; 1996. p. 335-343.

39. Connell RW. Gender and Power. Cambridge: Polity Press; 1987.

40. Connell RW. La organización social de la masculinidad. En: Valdés T, Olavarría J, editores. Masculinidades. Poder y crisis. Chile: Isis Internacional;1997. p. 31-48.

41. Courtenay WH. Constructions of masculinity and their influence on men's weell-being: a theory of gender and health. Social Science and Medicine. 2000;50:1385-1401.

42. Esteban ML. ¿Y los hombres? InguruakRevista Vasca de Sociología y Ciencia Política. 1998;22:15-29.
43. Otegui R. La construcción social de las masculinidades. Política y sociedad. 1999;32:151160.

44. Butler J. El género en disputa. México: Paidós; 2001.

45. Esteban ML. La antropología del cuerpo. Género, itinerarios corporales, identidad y cambio. Barcelona: Edicions Bellaterra; 2004.

46. Connell RW. Masculinities. Oxford, Cambridge: Polity Press; 1995. p. 45-67.

47. Esteban ML. Cuidado y salud: costes en la salud de las mujeres y beneficios sociales. En: Congreso Internacional Sare 2003. Cuidar cuesta: costes y beneficios del cuidado. Gasteiz: Emakunde-Instituto Vasco de la Mujer y Comunidad Europea/Fondo Social Europeo; 2004. p. 63-84.

48. Durán MA. Costes invisibles de la enfermedad. Bilbao: Fundación BBV; 1999.

49. Rodríguez Cabrero G, director. La protección social de la dependencia. Madrid: IMSERSO, Ministerio de Trabajo y Asuntos Sociales; 1999.

50. Comas d'Argemir D. Mujeres, familia y estado del bienestar. En: del Valle $\mathrm{T}$, editora. Perspectivas feministas desde la antropología social. Barcelona: Ariel; 2000. p. 187-204.

51. Menéndez EL. Hacia una práctica médica alternativa. Hegemonía y autoatención (gestión) en salud. Cuadernos de la casa chata.1983:86.

52. Menéndez EL. Grupo doméstico y proceso salud/enfermedad/atención: del teoricismo al movimiento continuo. Cuadernos Médico Sociales. 1992;59:3-18.

53. González Chévez L. El pulso de la sobrevivencia. Estrategias de atención para la salud en un colectivo de mujeres del subproletariado urbano. [Tesis doctoral] Universitat de Barcelona; 1998. [Inédita].

54. Osorio RM. Entender y atender la Enfermedad. Los saberes maternos frente a los padecimientos infantiles. México: CIESAS-INAH-INI; 1999.

55. Comelles JM. Sociedad, salud y enfermedad: los procesos asistenciales. Trabajo social y salud. 1998;29:135-150.

56. Colectivo Precarias a la Deriva. A la deriva por los circuitos de la precariedad femenina. Madrid: Proyecto Editorial Traficantes de Sueños; 2004.

57. Pérez Orozco A. Crisis de los cuidados: el sistema socioeconómico en reorganización. Hika. Septiembre 2005;169:24-26. 
58. Mauss M. Ensayo sobre los dones. Motivo y forma del cambio en las sociedades primitivas. En: Sociología y Antropología. 2a ed. Madrid: Tecnos; 1991. p. 153-263.

59. Shapiro J. Anthropology and the study of gender. En: Langland E, Gove W, editores. A Feminist Perspective in the Academy. Chicago: The University of Chicago Press; 1981. p. 110-129.
60. Maquieira V. El IUEM entre dos siglos: Lugares de la memoria y la acción. En: Maquieira $\mathrm{V}$, et al., editores. Democracia, feminismo y Universidad en el siglo XXI. 25 Aniversario del Instituto Universitario de Estudios de las Mujeres. Madrid: Instituto Universitario de Estudios de la Mujer; 2005. En prensa.

Recibido el 14 de septiembre de 2005

Versión final presentada el 14 de noviembre de 2005

Aprobado el 2 de diciembre de 2005 